\title{
Emprendimiento femenino y financiación social: un estudio comparado
}

\author{
Juan Carlos García Villalobos ${ }^{1}$, David Villaseca Morales², Susana González Pérez ${ }^{3}$
}

Recibido: 25 de marzo de 2019 / Aceptado: 4 de junio de 2019

Resumen. En el actual entorno económico mundial organismos como la Organización para la Cooperación y el Desarrollo Económico (OCDE) o el Banco Mundial reconocen el emprendimiento de nuevos negocios como un motor de crecimiento indiscutible y a los mecanismos financieros para ponerlo en práctica como determinantes. La literatura económica desde hace tiempo viene estudiando los procesos de emprendimiento desde diferentes perspectivas; aunque no tanto atendiendo al género de quien toma la iniciativa empresarial y a las singularidades de esta circunstancia, ya que hay situaciones que pueden llegar a condicionar la puesta en marcha de negocios por mujeres como el menor acceso a la financiación bancaria frente a sus homólogos masculinos, lo que puede justificar una menor actividad emprendedora. En ámbitos específicos como el emprendimiento social, donde el componente del lucro se ajusta con la dimensión social y medioambiental del proyecto, se pone de manifiesto esta situación. El presente artículo se centra en la demanda de financiación por parte de las emprendedoras a partir de tres bloques de análisis. En el primero, se estudian las necesidades de recursos financieros de las emprendedoras frente a los emprendedores con el propósito de establecer si hay diferencias relevantes, contrastando si las primeras precisan menos financiación que los segundos a la hora de llevar a cabo iniciativas empresariales. En el siguiente bloque, se analizan las fuentes de financiación principal declaradas por las emprendedoras de 69 países en la encuesta del Global Entrepreneurship Monitor (GEM), identificando varios clústeres relevantes con resultados diversos. Esto último hace posible, para terminar, contrastar empíricamente las diferencias en las fuentes principales expresadas por hombres y mujeres para financiar sus negocios ${ }^{4}$.

Palabras clave: Emprendimiento femenino; Mecanismos de financiación; Discriminación; Acceso a financiación; Emprendimiento social.

Claves Econlit: L26; M13.

\section{[en] Female entrepreneurship and social finance: a comparative study}

Abstract. In the current global economic environment, institutions such as the Organization for Economic Cooperation and Development (OECD) or the World Bank recognize the entrepreneurship

1 Universidad CEU San Pablo, España

Dirección de correo electrónico: garvil@ceu.es

2 Universidad CEU San Pablo, España

Dirección de correo electrónico: david.villasecamorales@colaborador.ceu.es

3 Universidad CEU San Pablo, España

Dirección de correo electrónico: susana.gonzalezperez@ceu.es

4 Este artículo forma parte de los trabajos vinculados al Proyecto de I+D+I FEM2017-83006-R, financiado por el Ministerio de Economía, Industria y Competitividad -Agencia Estatal de Investigación (AEI) y el Fondo Europeo de Desarrollo Regional (FEDER, UE). 
of new businesses as a driver of unquestionable growth, and the financial mechanisms to put it into practice as determinants. The economic literature has long been studying the processes of entrepreneurship from different perspectives; although not so much considering the gender of the entrepreneur and the singularities of this circumstance, since there are situations that can lead to condition the start-up of business by women as less access to bank financing compared to their male counterparts, which may justify a lower entrepreneurial activity. In specific fields like social entrepreneurship, where the profit component fits with the social and environmental dimension of the project, this situation can be shown. This article focuses on the demand for financing by female entrepreneurs on three blocks of analysis. In the first block, the needs of financial resources of female entrepreneurs are studied in comparison with male entrepreneurs, in order to establish if there are relevant differences, contrasting whether the former need less funding than the latter when carrying out business initiatives. In the next block, the main financing sources declared by female entrepreneurs of 69 countries in the Global Entrepreneurship Monitor (GEM) survey are analyzed, identifying several relevant clusters with diverse results. The latter makes it possible, in the end, to contrast empirically the differences in the main sources expressed by men and women to finance their businesses.

Keywords: Female entrepreneurship; Financing mechanisms; Discrimination; Access to financing; Social entrepreneurship.

Sumario. 1. Introducción: emprendimiento y género. 2. Claves en la financiación del emprendimiento. 3. Retos de oferta y demanda de financiación de emprendedoras. 4. Metodología y datos de la demanda para un estudio internacional. 5. Análisis internacional de las fuentes de financiación de las emprendedoras. 6. Conclusiones. 7. Referencias bibliográficas.

Cómo citar: García Villalobos, J.C., Villaseca Morales, D. y González Pérez, S. (2019) Emprendimiento femenino y financiación social: un estudio comparado. REVESCO. Revista de Estudios Cooperativos, Tercer Cuatrimestre, No 132, pp. 97-121. DOI: 10.5209/REVE.65974.

\section{Introducción: emprendimiento y género}

El fenómeno del emprendimiento empresarial es un tema de gran actualidad, tanto en la realidad económica mundial (European Parliament, 2015; OECD, 2017; World Bank, 2018) como en la literatura económica (Roper y Scott, 2009; Ruiz et al., 2010; Schoon y Duckworth, 2012; Ascher, 2012). En un contexto de desarrollo tras la crisis económica, la OCDE reconoce que la mayoría de los países están registrando crecimientos en la creación de nuevas empresas (OECD, 2017). En especial, destaca el aumento del número de empresas en el sector servicios por encima de otros sectores, ya que para el conjunto de países de la OCDE la creación de nuevas empresas en el sector servicios representa dos tercios de todos los empleos generados por el total de nuevas empresas.

La creación de empresas abre importantes oportunidades para mujeres de todo el mundo. Según el GEM, 163 millones de mujeres estaban iniciando o desarrollando nuevos negocios en todo el mundo y 111 millones estaban al frente de otros ya consolidados (GEM, 2017). En algunas economías estos negocios están asociados a actividades económicas básicas de autoempleo y el emprendimiento está asociado a la necesidad ${ }^{5}$. En otras economías más maduras, este

5 En economías como la India, el GEM identifica una actividad emprendedora básica donde aún tiene un alto peso la población rural. 
emprendimiento está asociado a la oportunidad que las emprendedoras ven en innovar creando nuevas start-ups ${ }^{6}$.

Sin embargo, estos datos pueden acompañar potenciales amenazas sociales en términos de discriminación de género. Aunque según la OCDE (OECD, 2017) las mujeres representan en torno a la mitad de la población mundial, sólo el 16\% de los fundadores de start-ups son mujeres. Conforme señala el GEM, las mujeres declaran globalmente una intención de emprender cercana a la declarada por los hombres. La intención promedia de emprender que muestran los datos del GEM es en todos los países encuestados de un $22 \%$ entre mujeres, y de un $29 \%$ entre los hombres. En los países europeos más enfocados en la innovación (como España ${ }^{7}$, Francia o Reino Unido) la intención media de emprendimiento de las mujeres es del $10 \%$, frente al $15 \%$ de los hombres. Por su parte, en los menos avanzados y más enfocados en la eficiencia (como Rusia, Polonia o Hungría) las mujeres declaran una intención del $15 \%$ frente al $24 \%$ de los hombres. A lo largo del proceso de emprendimiento, más mujeres que hombres no materializan esta intención en emprendimiento efectivo, decayendo en su intención a lo largo del camino. El Parlamento de la Unión Europea (European Parliament, 2015) reconoce que las mujeres representan sólo el 29\% de los 40,6 millones de emprendedores en el ámbito de la Unión Europea. Además, estas emprendedoras operan negocios más pequeños, dentro de sectores menos rentables financieramente y con un menor crecimiento que las empresas de sus homólogos masculinos (European Parliament, 2015). Por tanto, la situación tiene dos elementos de discusión: la creación de empresas por mujeres; por un lado, y su rentabilidad; por el otro.

En este sentido y en el caso del emprendimiento social español ${ }^{8}$, se evidencia una menor valoración de los proyectos desarrollados por mujeres por razón de la actividad económica llevada a cabo, dado que proliferan en el ámbito de la salud y la educación, con retornos más a largo plazo que los que pueden proporcionar los negocios de los hombres, más involucrados en ámbitos de contenido tecnológico y que presentan más escalabilidad e inmediatez en el retorno de la inversión (Buckland y Cordobés, 2017).

\section{Claves en la financiación del emprendimiento}

\subsection{La relevancia de la financiación para los emprendedores}

En cualquier emprendedor se presentan una serie de características que los definen: son capaces de detectar oportunidades de negocio, tienen la expectativa de éxito y asumen los riesgos de un contexto incierto (económicos, financieros, tecnológicos...). Pueden serlo tanto a título individual, normalmente como trabajador autónomo o por cuenta propia; o bien vinculado con otros mediante una

6 El GEM identifica un emprendimiento más enfocado en servicios, conocimiento e innovación en países como Estados Unidos, Francia, Reino Unido o España.

7 Montero y Camacho (2018) proponen un modelo para determinar las variables determinantes del emprendimiento femenino en España con base en base a los datos de la Encuesta de Población Activa de 2017.

8 Un estudio doctrinal y aplicado del emprendimiento social puede verse en: Sánchez Espada, J. et al (2018). 
personalidad jurídica o figura societaria concreta que le puede permitir trabajar en el negocio. Ante la importancia que adquiere el emprendimiento, organismos como la OCDE han analizado los factores que determinan la viabilidad de la intención de emprender. En su Entrepreneurship Indicators Programme (EIP) identifica seis determinantes que influyen en la actividad del emprendedor (OECD, 2017, p. 15):
1. El entorno regulatorio.
2. Las condiciones de mercado.
3. El acceso a financiación.
4. La creación y difusión de conocimiento.
5. La formación y capacidades.
6. La cultura.

En el acceso a la financiación recoge diversas fuentes posibles de financiación del emprendimiento:
- $\quad$ El endeudamiento.
- Los business angels.
- $\quad$ El capital-riesgo.
- $\quad$ El crowdfunding.
- $\quad$ La emisión de acciones.
- $\quad$ Los otros tipos de acceso a capital.

De esta forma, la OCDE reconoce la relevancia de la elección por los emprendedores entre las diferentes fuentes financieras, desde las más clásicas a otras más novedosas de captación de recursos.

\subsection{La financiación como barrera para los emprendedores}

El emprendimiento supone poner en marcha un proceso que conlleva actuar en diferentes ámbitos de gestión necesarios para consolidar una idea de negocio. Con frecuencia, esto supone hacer frente a limitaciones iniciales. Diferentes estudios han considerado los fundamentos para la viabilidad de la actividad emprendedora (Bates et al. 2007; Brush et al. 2009; Cohoon et al., 2010; Ascher, 2012). Bates et al (2007) proponen la teoría de las tres "Ms" sobre la creación y desarrollo de nuevas empresas. Estos tres ámbitos son: mercados ("market"), dinero ("money") y dirección ("management"). El mercado corresponde a la demanda destinataria de la producción, bien sea en mercados de consumo u organizacionales, el dinero a los recursos financieros necesarios y la dirección a la competencia a demostrar en la administración de cualquier negocio 9 .

En los últimos años se ha profundizado en las peculiaridades del emprendimiento femenino, destacando la importancia de la variable financiera (Brush et al, 2009; Henry et al., 2016). Los estudios económicos muestran como la financiación es la base para crear negocios innovadores y con potencial (Magri, 2009; Kwong et al. 2012). Pero, por otro lado, la financiación también puede convertirse en el principal freno al que se enfrentan las emprendedoras (Shaw et

9 Incluso se propone su evolución cuando se contempla desde el lado del emprendimiento femenino; un modelo ampliado de 5 "Ms" añade nuevas variables como la maternidad y el macro/micro-entorno. 
al., 2005; Méndez, 2010). Estos obstáculos pueden explicarse tanto desde el lado de la oferta de financiación, como desde el lado de la demanda. Así, por un lado, se han considerado posibles sesgos en el lado de la oferta de financiación por parte de los decisores de entidades financieras o de venture capital (Carter et al. 2007; Brush et al., 2009; Pogessi et al., 2016). En este sentido, diversos estudios demuestran como las prácticas de las entidades financieras (lado de la oferta) pueden generar obstáculos e insatisfacción en las emprendedoras (Carter, et al. 2007; Sena et al. 2012). Y, desde otro enfoque, se ha identificado la demanda de financiación de las propias emprendedoras como la limitación (Orser et al., 2006; Watson et al., 2017): las emprendedoras pueden preferir evitar la financiación social ajena para mantener el control de su negocio, o por aversión al riesgo, dado la alta intensidad del mismo que perciben (Marlow, Shaw y Carter, 2008). Esto se constata en el emprendimiento social español, donde la aversión a la solicitud de financiación ajena por emprendedoras les lleva a optar por reducir el tamaño del negocio o incluso ralentizar su crecimiento (Buckland y Cordobés, 2017).

El Parlamento de la Unión Europea recoge que las mujeres emprenden con menor capital inicial que los hombres, pero cuando lo hacen, acuden a fuentes informales como la familia en vez de acudir al mercado financiero (European Parliament, 2015). Además, matiza que esta situación de menor inversión y menor apalancamiento hace que las entidades financieras puedan esperar menores retornos, $y$, por tanto, mostrar un menor compromiso con sus negocios. La menor necesidad tanto de capital como de financiación por las emprendedoras frente a los emprendedores ha sido corroborada por la literatura bien por el menor tamaño de los negocios que crean, bien por las necesidades asociadas al sector o bien por actitudes frente al riesgo y el crecimiento (Marlow y Patton, 2005).

Desde un punto de vista cuantitativo, la encuesta de la OCDE sobre el emprendimiento (OECD, 2017) muestra las diferencias de género en términos de acceso a financiación. Analiza el porcentaje de mujeres y hombres que declaran, en cada país, que tienen acceso al dinero para iniciar o hacer crecer un negocio. En la mayoría de los países analizados por la OCDE, las mujeres declaran en menor medida que los hombres que puedan acceder a dicha financiación. En España, sólo el $18,5 \%$ de las mujeres declaran tener ese acceso, frente al 24,3\% de los hombres; en Francia, el 16,9\% de las mujeres frente al 26,7\% de los hombres; en Reino Unido, el 27,4\% de las mujeres frente al 46,4\% de los hombres. Como excepción, los dos países donde las mujeres declaran en mayor medida que los hombres tener acceso a la financiación para emprender son: Estados Unidos $(37,0 \%$ de las mujeres, frente al 35,3\% de los hombres) y México (17,2\% de las mujeres, frente al $16,4 \%$ de los hombres).

\subsection{Alternativas de fuentes de financiación para los emprendedores}

Los emprendedores pueden optar entre financiar su nuevo negocio con sus recursos propios, ampliando el capital o endeudándose acudiendo a entidades financieras o a otras fuentes. En un primer momento, el emprendedor social puede recibir el apoyo económico de personas cercanas como amigos y familiares mediante recursos que pueden articularse como préstamos o en forma de participación en el capital de la 
sociedad. Estas son una fuente informal y ágil, aunque limitada, de obtener los primeros recursos para emprender (Villaseca, 2014).

Más recientemente han surgido figuras financieras que se van consolidando en el mercado financiero: los "business angels" o mentores inversores, individuos con contactos, capacidad de inversión y conocimientos para apoyar el crecimiento del proyecto empresarial. Por su parte, el capital-riesgo lo componen compañías inversoras profesionales con mayores recursos y experiencia para desarrollar los nuevos negocios a través de un mayor control del proyecto a cambio de sus mayores aportaciones. Este mayor control configura el denominado capital-riesgo social donde lo que determina la decisión de inversión es el impacto social de la actividad financiada. Esto entraría dentro de lo que se denomina financiación solidaria junto con la banca ética, los prestamos sociales P2P o los bancos de tiempo, entre otros.

\section{Retos de oferta y demanda de financiación de emprendedoras}

\subsection{Indicios de discriminación de género en el lado de la oferta de financiación}

En la literatura económica (Verheul y Thurik, 2001; Marlow y Patton, 2005; Coleman et al., 2016), se analiza la dinámica de financiación de las emprendedoras, aunque desde parcelas específicas de esta realidad y en muchas ocasiones desde el lado de la oferta de financiación (Harrison y Mason, 2007). Algunos trabajos han prestado especial atención a la concesión de préstamos por bancos y otras entidades análogas (Carter et al, 2007). En términos generales, se consideran diferentes fuentes posibles de discriminación por el lado de la oferta (Hertz, 2011): porque las entidades financieras disuaden a las mujeres de solicitar préstamos, porque les ofrecen peores condiciones que a sus homólogos masculinos, o porque reciben condiciones poco ventajosas en los préstamos concedidos. Estos estudios generalmente están enfocados en posibles discriminaciones en el lado de la oferta de financiación, y no tanto en el comportamiento que subyace cuando se concreta la demanda de financiación por parte de las emprendedoras.

Los análisis de prácticas discriminatorias en el acceso a la financiación llegan al tipo de recurso utilizado, por ejemplo, diferentes estudios han analizado posibles comportamientos discriminatorios en el apoyo financiero de las empresas de venture capital (Brush et al., 2006; Tinkler et al., 2015). El género del emprendedor puede resultar relevante en las decisiones de estas empresas cuando pesa más la evaluación de la persona que la de la propia empresa, aunque esto puede a su vez verse atenuado gracias a la formación técnica o la cercanía al equipo de la empresa de venture capital (Lins y Lutz, 2016).

Del mismo modo, se ha profundizado en el efecto del género en la demanda de financiación mediante fuentes masivas de potenciales inversores sociales, esto es, el crowdsourcing (Marom et al., 2016). Con base en el análisis de plataformas de financiación masivas como Kickstarter, se confirma que las mujeres piden menos volúmenes de capital. En consecuencia, consiguen menos financiación: sólo el 23\% de los proyectos financiados por inversores hombres estaban liderados por mujeres, 
cifra que ascendía al $40 \%$ entre cuando las inversoras eran mujeres ${ }^{10}$; sin embargo, resulta difícil conocer el peso del componente social del proyecto en las decisiones de estos inversores.

\section{2. ¿Segundo techo de cristal? El lado de la demanda de financiación}

La realidad mencionada hace que los obstáculos para el emprendimiento femenino puedan considerarse para las mujeres un "segundo techo de cristal" (Bosse et al., 2012), de forma análoga a las limitaciones que las mujeres pueden encontrar para ascender en el entorno laboral (Cotter et al., 2001), o ser miembros del Consejo de Administración de grandes empresas (del Cabo et al., 2011). Este segundo techo de cristal se refiere al sesgo de género que impediría que nuevas empresas fundadas por mujeres puedan acceder al capital financiero necesario para su desarrollo y crecimiento (Bosse et al., 2012).

Sin embargo, estos estudios no profundizan tanto en el lado de la demanda de capital o deuda por parte de las emprendedoras. Siguiendo el modelo de Pecking Order Theory, las asimetrías de información sobre las perspectivas de sus nuevos negocios harían que primero los emprendedores prefieran la financiación propia, luego deuda, y finalmente capital externo (Myers, 1984). Estas preferencias afectarían al resultado final de financiación de los recursos necesarios para emprender. Se abren así nuevas líneas de investigación que contrastan la discriminación de género respecto a una falta de inclinación a pedir financiación por parte de las emprendedoras (Sena et al., 2012; Watson et al. 2017) ${ }^{11}$.

\section{Metodología y datos de la demanda para un estudio internacional}

\subsection{Hipótesis y metodología de análisis}

Ante la importancia de entender las dinámicas en la demanda de financiación por parte de las emprendedoras, se propone un análisis empírico sobre datos de encuestas de diferentes países en este colectivo. En primer lugar, se desarrolla un análisis descriptivo para identificar las necesidades de financiación de las emprendedoras en comparación con sus homólogos varones. Diversos autores han defendido su menor necesidad de capital (Marlow y Swail, 2014; European Parliament 2015). De esta forma se confirmará si las necesidades declaradas de capital para emprender son similares o si varían en función del género, y si esto es homogéneo en los diferentes países. Esto se concreta en una primera hipótesis a contrastar:

10 Similares estudios se han realizado sobre el apoyo de business angels (Harrison y Mason, 2007; Lange et al., 2007).

11 No obstante, hay estudios que recogen esta visión de la demanda mediante datos declarados por los emprendedores, como la encuestas Kauffman Firm Survey en Estados Unidos (Coleman y Robb, 2009), o en ámbito internacional las encuestas de GEM-Global Entrepreneurship Monitor (Roper y Scott, 2009). En España, también se han utilizado datos de GEM-Global Entrepreneurship Monitor para entender estas dimensiones del emprendimiento (Ruiz et. al, 2009; Mendez, 2010). 
"Hipótesis 1: Las emprendedoras (mujeres) necesitan la misma cantidad dinero para emprender sus negocios que los emprendedores (hombres)."

A partir de aquí, el análisis de clústeres de K-medias permitirá crear grupos homogéneos de países según las fuentes preferidas por las emprendedoras para financiar sus negocios, de forma que se clasifiquen a los diferentes países según las fuentes de financiación principal para el nuevo negocio. Estos clústeres mostrarán, en su caso, si existen particularidades nacionales que determinen la preferencia por uno u otro tipo de financiación, así como la importancia del contexto en su elección. Por tanto, se contemplará la segunda hipótesis:

"Hipótesis 2: Las preferencias de las emprendedoras (mujeres) sobre las fuentes para financiar sus negocios no varían según el país donde desarrollan su actividad."

Finalmente, se analizará mediante pruebas de chi-cuadrado las respuestas de emprendedoras y emprendedores de los países analizados, para determinar una cuestión clave en el emprendimiento en términos de género: si la preferencia de fuentes de financiación de las mujeres es diferente a la de los hombres. Diversos estudios han afirmado una menor tendencia en las mujeres a fuentes de financiación ajena de bancos y otras instituciones (Coleman y Robb, 2009; Xiong et al., 2011).

"Hipótesis 3: Las emprendedoras (mujeres) prefieren financiar su negocio con las mismas fuentes de financiación que los emprendedores (hombres)"

\subsection{Fuentes de información del estudio}

Para desarrollar estos análisis, se utiliza la información internacional de las encuestas desarrolladas por el GEM-Global Entrepreneurship Monitor para 2014. A través de un consorcio de equipos de investigación nacionales, este estudio desarrolla encuestas en más de sesenta países, con muestras de al menos 2.000 adultos en edad de trabajar en cada país, entre 18 y 65 años $^{12}$.

La ventaja de este estudio a nivel de población activa es que permite entender las actitudes y comportamientos ante el emprendimiento en sus diferentes fases:

- $\quad$ Los potenciales emprendedores en fases previas del emprendimiento.

- $\quad$ Los que tienen intención de emprender en los próximos tres años.

- $\quad$ Los que efectivamente están creando un nuevo negocio.

- Los nuevos emprendedores con un negocio que ya está operando, entre tres y 42 meses.

- Los empresarios ya establecidos con negocios maduros de más de 42 meses.

- $\quad$ Los que han abandonado en el último año su negocio.

12 La investigación cubre amplias áreas geográficas: Asia-Pacífico; Europa y Asia Central; Latino América y Caribe, Norte América, Oriente Medio y África. Las encuestas, Adult Population Survey, se desarrollan con muestras aleatorias representativas de la población, con un cuestionario estándar para lograr resultados comparables. En todo caso, el equipo global de GEM asegura la calidad del estudio en cada país, así como la comparación entre países. 
Esta fuente de información ha sido empleada en diferentes estudios para analizar la realidad del emprendimiento femenino (Ruiz et al., 2010; Méndez, 2010), pero en menor medida para profundizar en los retos de la demanda de financiación por las emprendedoras (Roper, 2009).

Además, se completará este estudio con datos macroeconómicos del Banco Mundial (World Bank, 2018) y la OCDE (OECD, 2017) con el fin de situar el contexto económico de los diferentes países. De esta forma se interpretarán los análisis considerando la realidad local (por ejemplo, en términos de desarrollo económico nacional o situación laboral de las mujeres). Con esto se pretende explicar la relevancia del sistema financiero doméstico a la hora de condicionar las expectativas de acceso a financiación.

\subsection{Colectivo muestra de análisis}

En esta parte, se analizan las diferencias declaradas en las expectativas de recursos financieros necesarios para un nuevo negocio en la encuesta de GEM (Global Entrepreneurship Monitor, 2017). La muestra de análisis serán aquellos individuos que muestran alguna actividad de emprendimiento, tanto en fase potencial, como de inicio de actividad o efectivamente en fase de desarrollo de su negocio. Se excluyen así los individuos que no desarrollan actividades de emprendimiento activamente. Y como segundo criterio de filtro, se establece que además contemplen que su actividad requiere de algún tipo de financiación. Se excluyen así las iniciativas donde los emprendedores declaran expresamente no requerir de ningún tipo de capital ni financiación.

El colectivo así definido, individuos con actividad emprendedora y requerimientos financieros, limita de una forma relevante la muestra a analizar, pero es crítico para acotar los resultados a la realidad de los emprendedores. Así, en el año 2014 estudiado, los datos disponibles en las encuestas en 69 países del GEM ofrecen un total de más de 200.000 encuestados, adultos en edad de trabajar. Esta muestra se reduce a 16.920 cuando filtramos las declaraciones de los emprendedores para representar este colectivo.

Dentro de este grupo, se crearán además dos grupos diferenciados en función del género que declaran: hombres y mujeres. Como es de esperar, a causa de la menor tasa de emprendimiento femenina, el porcentaje de mujeres que se consideran en alguna fase de emprendimiento es inferior a la de los hombres: el $6,8 \%$ del total de las mujeres encuestadas se incluyen en el grupo objeto de estudio; frente al $10,1 \%$ de los hombres encuestados. Este primer dato ratifica los datos de menor emprendimiento femenino que recogen organismos internacionales (European Parliament, 2015; OECD, 2017). 


\section{Análisis internacional de las fuentes de financiación de las emprendedoras}

\subsection{Análisis de las necesidades de financiación de los emprendedores en función de su genero}

Para contrastar la primera hipótesis, comparamos las diferencias entre las cantidades que los emprendedores declaran como requeridas para iniciar su negocio. En este caso se analizan los principales países de la zona Euro: Austria, Bélgica, Alemania, España, Finlandia, Francia, Grecia, Irlanda, Italia, Holanda y Portugal.

Los datos de la encuesta de GEM del año 2014 muestran fuertes diferencias regionales en las cantidades de inversión estimadas por los emprendedores en su conjunto. En países como España, Grecia o Finlandia, los emprendedores (hombres y mujeres) estiman necesitar menos de 100.000 euros para su nuevo negocio. En concreto, los emprendedores en España declaran la menor cantidad los países analizados, con una media de 56.000 euros. En el resto de países, las estimaciones de recursos necesarios declaradas exceden los 100.00 euros.

En términos de género, los datos (Tabla 1) muestran que, entre los once países analizados, en el $64 \%$ las emprendedoras estiman necesitar de media menos dinero que sus homólogos masculinos. Esto es consistente con la literatura económica que afirma menores necesidades de dinero por parte de las mujeres que emprenden (Marlow y Patton, 2005), así como con las tesis de diferentes organismos oficiales (European Parliament, 2015). En el caso de España, las emprendedoras declaran necesitar de media 50.500 euros para su nuevo negocio, frente a los 61.400 de los emprendedores (hombres). Esta pauta no se contrasta en todos los países analizados. En el 36\% de los países analizados, la cantidad de dinero requerida para iniciar un negocio es mayor en las emprendedoras (caso de Bélgica, Finlandia, Holanda y Portugal). 
Tabla. 1. Cantidad de recursos financieros requeridos por los emprendedores para su negocio

\begin{tabular}{|c|c|c|c|c|c|}
\hline \multicolumn{2}{|c|}{$\begin{array}{l}\text { ¿Cuánto dinero, en total, será requerido para } \\
\text { empezar el nuevo negocio? }\end{array}$} & $\mathrm{N}$ & \multirow{2}{*}{$\frac{\text { Media }}{228.319}$} & \multirow[t]{2}{*}{$\begin{array}{c}\begin{array}{c}\text { Diferencia Mujeres- } \\
\text { Hombres }\end{array} \\
\end{array}$} & \multirow[t]{2}{*}{$\begin{array}{c}\text { Prueba } t \text { igualdad de medias/ } \\
\text { Sig. (bilateral) } 90 \% \text { / No se } \\
\text { asumen Var iguales }\end{array}$} \\
\hline AT & Hombre & 99 & & & \\
\hline & Mujer & 50 & 61.458 & 166.861 & 0,12 \\
\hline \multirow[t]{2}{*}{$\mathrm{BE}$} & Hombre & 46 & 88.768 & & \\
\hline & Mujer & 13 & 147.554 & 58.785 & 0,56 \\
\hline \multirow[t]{2}{*}{$\mathrm{DE}$} & Hombre & 101 & 358.852 & & \\
\hline & Mujer & 55 & 136.444 & 222.409 & 0,08 \\
\hline \multirow[t]{2}{*}{ ES } & Hombre & 298 & 61.421 & & \\
\hline & Mujer & 150 & 50.589 & 10.832 & 0,32 \\
\hline \multirow[t]{2}{*}{$\mathrm{FI}$} & Hombre & 26 & 54.982 & & \\
\hline & Mujer & 14 & 95.464 & 40.483 & 0,59 \\
\hline \multirow[t]{2}{*}{ FR } & Hombre & 29 & 1.411 .010 & & \\
\hline & Mujer & 16 & 80.350 & 1.330 .660 & 0,30 \\
\hline \multirow[t]{2}{*}{ GR } & Hombre & 35 & 113.286 & & \\
\hline & Mujer & 11 & 49.591 & 63.695 & 0,12 \\
\hline \multirow[t]{2}{*}{ IE } & Hombre & 59 & 6.104 .669 & & \\
\hline & Mujer & 24 & 462.646 & 5.642 .024 & 0,22 \\
\hline \multirow[t]{2}{*}{ IT } & Hombre & 31 & 648.174 & & \\
\hline & Mujer & 15 & 29.267 & 618.908 & 0,21 \\
\hline \multirow[t]{2}{*}{ NL } & Hombre & 52 & 120.954 & & \\
\hline & Mujer & 30 & 538.520 & 417.566 & 0,41 \\
\hline \multirow[t]{2}{*}{ PT } & Hombre & 67 & 88.820 & & \\
\hline & Mujer & 43 & 155.558 & 66.738 & 0,58 \\
\hline
\end{tabular}

(Fuente: Elaboración propia, con base en Encuesta GEM 2014)

Sin embargo, todas estas diferencias de las medias declaradas por hombres y mujeres no son estadísticamente significativas ${ }^{13}$. En consecuencia, no podemos rechazar la hipótesis 1, "Las emprendedoras (mujeres) necesitan la misma cantidad dinero para emprender sus negocios que los emprendedores (hombres)". Frente a la literatura económica que afirma una menor necesidad de dinero de las emprendedoras (Marlow y Patton, 2005; Coleman y Robb, 2009), este análisis en el ámbito de países de la zona Euro no avala que las mujeres necesiten menos dinero que los hombres. La ventaja de contar con una fuente de información declarativa es que refleja las intenciones de los emprendedores, incluso antes de encontrar posibles obstáculos en el proceso de obtención de financiación. En consecuencia, resulta importante entender qué fuentes buscan las emprendedoras para satisfacer esas necesidades económicas. Además, las diferencias expresadas cuando comparamos los diferentes países confirman la necesidad de contemplar las

13 Para un nivel de confianza del $95 \%$ y dado que el p-valor supera en todos los casos el 0,05. 
peculiaridades regionales en el estudio de la demanda de financiación por parte de las emprendedoras.

\subsection{Clústeres de países en función de las fuentes de financiación preferidas por las emprendedoras}

Para contrastar la segunda hipótesis, partimos de los datos manifestados por los emprendedores, hombres y mujeres, para los 69 países que recoge la encuesta del GEM. Éstos indican el origen del dinero que necesitan para emprender su nuevo negocio. Las posibles respuestas como fuentes de financiación son cinco: a) sus propios ahorros; b) los ahorros de su familia; c) los bancos $\mathrm{u}$ otras instituciones financieras; d) los amigos y e) otros. No obstante, no detalla las nuevas fuentes de financiación que emergen en los países más innovadores, como business angels o venture capital (Villaseca, 2014), que quedarían agrupados en el concepto de "otros".

La segmentación realizada ha permitido recoger esos 69 países contemplados en el GEM en cinco clústeres (Cuadro 1). El clúster número 3 es el más numeroso, ya que recoge 28 países; mientras que el clúster 2 contiene 11 países y los restantes tres clústeres (clústeres 1,4 y 5) 10 países cada uno.

Cuadro. 1. Clústeres de países según fuentes financiación principal de emprendedoras

\begin{tabular}{|l|l|}
\hline Cluster 1 & $\begin{array}{l}\text { Canadá, Bélgica, Francia, Noruega, Polonia, Colombia, Barbados, } \\
\text { Bostwana, El Salvador y Puerto Rico }\end{array}$ \\
\hline Cluster 2 & $\begin{array}{l}\text { Perú, Guatemala, Panamá, Bolivia, Ecuador, Irán, Kazajistán, } \\
\text { Burkina Faso, Kosovo, Croacia, y Bosnia. }\end{array}$ \\
\hline Cluster 3 & $\begin{array}{l}\text { Estados Unidos, México, Rusia, España, Portugal, Holanda, } \\
\text { Rumanía, Austria, Alemania, Suecia, Luxemburgo, Irlanda, } \\
\text { Finlandia, Estonia, Eslovenia, Eslovaquia, Argentina, Brasil, } \\
\text { Chile, Costa rica, Uruguay, Belice, Malasia, Angola, Qatar, } \\
\text { Trinidad y Tobago, Jamaica y Sudáfrica. }\end{array}$ \\
\hline Cluster 4 & $\begin{array}{l}\text { India, China, Filipinas, Tailandia, Vietnam, Grecia, Hungría, } \\
\text { Italia, Lituania y Surinam }\end{array}$ \\
\hline Cluster 5 & $\begin{array}{l}\text { Australia, Japón, Indonesia, Singapur, Taiwán, Reino Unido, } \\
\text { Suiza, Dinamarca, Camerún y Uganda }\end{array}$ \\
\hline
\end{tabular}

Fuente: Elaboración propia, sobre datos GEM.

La técnica que permite contrastar el sesgo nacional en las respuestas dadas por las emprendedoras es la prueba Chi-cuadrado entre las respuestas de fuentes de financiación de los 69 países citados más arriba (Tabla 2). Se confirma las diferencias nacionales en las respuestas dadas por las emprendedoras. Para profundizar en estas diferencias, el siguiente estudio desarrollado para agrupar los países en función de las fuentes de financiación declaradas por las emprendedoras es un análisis de clústeres de K-medias. Para explicar los diferentes clústeres son necesarias varias de las fuentes de financiación posibles (Tabla 3), lo que confirma 
la diversidad de preferencias. Sólo hay una fuente, la financiación de amigos, que no resulta significativa a la hora de agrupar estos clústeres: en muchos países ni siquiera consideran las emprendedoras esta fuente de financiación.

Tabla. 2. Prueba Chi-cuadrado por países según fuentes financiación declaradas por emprendedoras

\begin{tabular}{|l|r|r|r|}
\hline \multicolumn{2}{|c|}{ Pruebas de chi-cuadrado } & $\begin{array}{c}\text { Significación } \\
\text { asintótica } \\
\text { (bilateral) }\end{array}$ \\
\hline Chi-cuadrado de Pearson & Valor & df & 0,000 \\
\hline Razón de verosimilitud & $1351,075^{a}$ & 276 & 0,000 \\
\hline Asociación lineal por lineal & 1321,355 & 1 & 0,000 \\
\hline N de casos válidos & 16,266 & & \\
\hline
\end{tabular}

Fuente: Elaboración propia, sobre Encuesta GEM 2014.

Tabla. 3. Centros de Clústeres de países según fuentes financiación declaradas por emprendedoras

\begin{tabular}{|l|c|c|c|c|r}
\hline \multicolumn{7}{|c|}{ Centros de clústeres finales } \\
\hline & 1 & 2 & \multicolumn{3}{c}{ Clúster } \\
\cline { 2 - 7 } & 0,4710 & 0,3536 & 0,5446 & 0,3750 & 0,7240 \\
\hline Ahorros Personales & 0,0850 & 0,2355 & 0,1546 & 0,3810 & 0,1080 \\
\hline Ahorros Familia & 0,3260 & 0,3636 & 0,2096 & 0,1610 & 0,1160 \\
\hline $\begin{array}{l}\text { Bancos y otras } \\
\text { Instituciones Fin. }\end{array}$ & 0,0180 & 0,0173 & 0,0150 & 0,0300 & 0,0230 \\
\hline Amigos & 0,1010 & 0,0291 & 0,0761 & 0,0530 & 0,0290 \\
\hline Otros & & & & & \\
\hline
\end{tabular}

\begin{tabular}{|c|c|c|c|c|c|c|}
\hline \multicolumn{7}{|c|}{ ANOVA } \\
\hline & \multicolumn{2}{|c|}{ Clúster } & \multicolumn{2}{|c|}{ Error } & \multirow[b]{2}{*}{$\mathrm{F}$} & \multirow[b]{2}{*}{ Sig. } \\
\hline & $\begin{array}{c}\text { Media } \\
\text { cuadrática }\end{array}$ & gl & $\begin{array}{c}\text { Media } \\
\text { cuadrática }\end{array}$ & gl & & \\
\hline Ahorros Personales & 0,239 & 4 & 0,004 & 64 & 68,077 & 0,000 \\
\hline Ahorros Familia & 0,149 & 4 & 0,004 & 64 & 38,112 & 0,000 \\
\hline $\begin{array}{l}\text { Bancos y otras } \\
\text { Instituciones Fin. }\end{array}$ & 0,119 & 4 & 0,004 & 64 & 31,009 & 0,000 \\
\hline Amigos & 0,000 & 4 & 0,000 & 64 & 1,347 & 0,262 \\
\hline Otros & 0,011 & 4 & 0,002 & 64 & 4,715 & 0,002 \\
\hline
\end{tabular}

Fuente: Elaboración propia, sobre Encuesta GEM 2014. 
El detalle de los centros de los clústeres (Tabla 3) muestra importantes diferencias entre ellos. Así, el clúster con más países, el 3, es el que muestra la preferencia tanto de fuentes de financiación propias (ahorro personal), como ajenas. Las emprendedoras de los países del clúster 3 declaran como principales varias fuentes principales de dinero para su nuevo negocio. Consideran que el dinero requerido procederá en gran medida de ahorros personales, y también consideran muchas de ellas como importante la financiación externa, bien de bancos y entidades financieras, o bien de su propia familia. Incluso consideran que el grupo de otras fuentes será relevante.

Los clústeres 1 y 2 agregan países donde, comparativamente, las emprendedoras cuentan más con la financiación ajena de bancos e instituciones financieras. En concreto, el clúster 1 se caracteriza por una relevancia de ahorros personales seguida de la financiación mediante bancos e instituciones financieras. Incluso en este clúster adquiere cierta relevancia de financiación mediante otras fuentes. Por su parte, el clúster 2 se caracteriza por la mayor relevancia de la financiación ajena, que se consigue principalmente a través de los bancos, pero también informalmente gracias a ahorros de familiares. En términos de la clasificación que GEM hace sobre el nivel de avance de cada economía, estos países del clúster 2 tienen en común su caracterización como países con un nivel de desarrollo medio o bajo.

Los clústeres 4 y 5 destacan por el menor peso de la financiación formal a través de bancos e instituciones financieras. Estos clústeres son especialmente relevantes en las economías de Asia-Pacífico. En concreto, la fuente principal de financiación en los países del clúster 4 es la aportada por los propios familiares de la emprendedora. Nuevamente, estos países que se apoyan más en la financiación de familiares se encuadran en niveles de desarrollo económico medio o bajo, según la clasificación de GEM (Global Entrepreneurship Monitor, 2017), con la excepción de Italia y Grecia (que se clasifican en el nivel superior de desarrollo). En cambio, en el clúster 5 la fuente de financiación dominante sería mayoritariamente los ahorros personales de la propia emprendedora, siendo residual la financiación ajena.

Con base en estos análisis (Tablas 2 y 3), podemos rechazar la hipótesis 2: "Las preferencias de las emprendedoras (mujeres) sobre las fuentes para financiar sus negocios no varían según el país donde desarrollan su actividad". Se ha podido confirmar la relevancia del contexto nacional en las preferencias de fuentes de financiación por parte de las emprendedoras. Los análisis muestran que hay diferentes preferencias de financiación según los países. También refuerza la importancia de profundizar en los análisis locales, no pudiendo generalizar sobre las preferencias de las emprendedoras sin tener en cuenta su contexto.

\subsection{Comparativa global de fuentes de financiación preferidas según el género de los emprendedores}

Tras los anteriores resultados, la cuestión que surge es si estas preferencias declaradas por las emprendedoras sobre sus fuentes de financiación están meramente determinadas por la geografía, o si además está condicionada por su género. Mediante pruebas de chi-cuadrado podemos contrastar si las preferencias 
declaradas por las emprendedoras son similares o diferentes a las declaradas por sus homólogos masculinos, en línea con la hipótesis 3.

En primer lugar, analizamos los datos de declarados de fuentes de financiación de emprendedoras frente a emprendedores, de forma agregada de los 69 países (Tabla 4).

Tabla. 4. Fuentes financiación declaradas por emprendedores, agregados 69 países

\begin{tabular}{|l|r|r}
\hline & Hombres & \multicolumn{1}{l}{ Mujeres } \\
\hline Ahorros Personales & $48,9 \%$ & $49,3 \%$ \\
\hline Ahorros Familia & $14,1 \%$ & $19,0 \%$ \\
\hline $\begin{array}{l}\text { Bancos y otras } \\
\text { Instituciones Fin. }\end{array}$ & $27,7 \%$ & $24,7 \%$ \\
\hline Amigos & $2,8 \%$ & $2,0 \%$ \\
\hline Otros & $6,5 \%$ & $5,0 \%$ \\
\hline Total & $100,0 \%$ & $100,0 \%$ \\
\hline
\end{tabular}

Fuente: Elaboración propia, sobre Encuesta GEM 2014.

La primera fuente declarada de financiación es común en ambos grupos: el $48,9 \%$ de los hombres y el 49,3\% de las mujeres declaran que la fuente principal de los fondos necesarios para su negocio serán sus propios ahorros. Sin embargo, las dos siguientes fuentes de financiación por importancia presentan diferencias en función del género. Los varones emprendedores en esta visión global declaran en mayor medida la financiación ajena a través de bancos y otras entidades financieras, con un $27,7 \%$ de las respuestas. Por su parte, las emprendedoras declaran esta fuente en el $24,7 \%$ de los casos. En cambio, las emprendedoras acudirán más a los ahorros de su familia, con un $19,0 \%$ de las respuestas, respecto a los emprendedores que sólo acuden a esta fuente en un 14,1\% de las ocasiones. Asimismo, el peso que cobran las otras formas de financiación es diferente: el 6,5\% de emprendedores acuden a estas fuentes alternativas que según se trató más arriba cobran peso en geografías como Estados Unidos o Europa. En el caso de las emprendedoras, esta cifra se reduce al $5 \%$.

Las pruebas de Chi-cuadrado confirman que las diferencias en estas cifras en función del género son significativas, por lo que a nivel agregado de los 69 países podemos rechazar la hipótesis 3: "Las emprendedoras (mujeres) prefieren financiar su negocio con las mismas fuentes de financiación que los emprendedores (hombres)". Este resultado confirmaría las diferentes teorías que señalan las diferentes preferencias que hombres y mujeres tienen a la hora de buscar financiación para un nuevo negocio (Sena et al., 2012; Watson et al. 2017). 


\subsection{Comparativa local de fuentes de financiación preferidas según el género de los emprendedores}

A pesar de los resultados globales, se ha comprobado con anterioridad las grandes diferencias que existen entre países en términos del fenómeno del emprendimiento. Esto hace necesario considerar bajo una dimensión nacional la comparativa entre hombres y mujeres que emprenden. Para ello, se lleva a cabo el mismo contraste entre mujeres y hombres dentro de los diferentes países para verificar si este resultado global se repite localmente. Empezando por España, los resultados locales (Tabla 5) muestran que las emprendedoras españolas se comportan de forma diferente que sus homólogos masculinos.

Tabla. 5. Fuentes financiación declaradas por emprendedores, España

\begin{tabular}{|l|r|r}
\hline & Hombres & Mujeres \\
\hline Ahorros Personales & $54,0 \%$ & $52,2 \%$ \\
\hline Ahorros Familia & $9,9 \%$ & $13,2 \%$ \\
\hline $\begin{array}{l}\text { Bancos y otras } \\
\text { Instituciones Fin. }\end{array}$ & $21,9 \%$ & $27,8 \%$ \\
\hline Amigos & $2,6 \%$ & $0,5 \%$ \\
\hline Otros & $11,5 \%$ & $6,3 \%$ \\
\hline Total & $100,0 \%$ & $100,0 \%$ \\
\hline
\end{tabular}

\begin{tabular}{|c|c|c|c|}
\hline \multicolumn{4}{|c|}{ Pruebas de chi-cuadrado } \\
\hline & Valor & df & $\begin{array}{c}\text { Significación } \\
\text { asintótica } \\
\text { (bilateral) }\end{array}$ \\
\hline Chi-cuadrado de Pearson & $10,148^{\mathrm{a}}$ & 4 & 0,038 \\
\hline
\end{tabular}

Fuente: Elaboración propia, sobre Encuesta GEM 2014.

Aunque su primera fuente de financiación será nuevamente los ahorros personales, declaran acudir a esta fuente en menor medida que los hombres: 52,2\% de mujeres frente a un $54.0 \%$ de hombres. A diferencia de los datos globales, los emprendedores españoles acuden más a esta fuente que las emprendedoras. Esto se explica porque las mujeres acuden en mayor medida, $27,8 \%$, que los hombres, $21,9 \%$, a la financiación bancaria. Otra importante diferencia entre ambos grupos es su consideración de las otras fuentes a su alcance, porque pues casi el doble de porcentaje de hombres $(11,5 \%)$ que de mujeres $(6,3 \%)$ lo considera su fuente principal de financiación. La prueba de chi-cuadrado en los datos de España nuevamente permite rechazar la hipótesis 3 , porque las emprendedoras prefieren distintas fuentes de financiación que sus homólogos masculinos. 
En otros países se contrastan resultados similares, dado que los datos permiten rechazar la hipótesis 3, es decir, se contrastan diferentes preferencias de fuentes de financiación según el género. Dentro del mismo clúster 3 donde se incluía España, encontramos países como Sudáfrica (Tabla 6), donde también contrastamos diferencias relevantes entre las fuentes declaradas por hombres y mujeres para sus nuevos negocios. En este país, las emprendedoras confían masivamente en los ahorros propios, con un $60 \%$, frente al $41,1 \%$ de los hombres, quienes a su vez confían en gran medida en bancos: $34,4 \%$ frente al 12,7 de las mujeres.

Tabla. 6. Fuentes financiación declaradas por emprendedores, Sudáfrica

\begin{tabular}{|c|c|c|}
\hline & Hombres & Mujeres \\
\hline Ahorros Personales & $41,1 \%$ & $60,0 \%$ \\
\hline Ahorros Familia & $14,4 \%$ & $18,2 \%$ \\
\hline $\begin{array}{l}\text { Bancos y otras } \\
\text { Instituciones Fin. }\end{array}$ & $34,4 \%$ & $12,7 \%$ \\
\hline Amigos & $2,2 \%$ & $0,0 \%$ \\
\hline Otros & $7,8 \%$ & $9,1 \%$ \\
\hline Total & $100,0 \%$ & $100,0 \%$ \\
\hline \multicolumn{3}{|c|}{ Pruebas de chi-cuadrado } \\
\hline Valor & \multirow[t]{2}{*}{ df } & $\begin{array}{l}\text { Signinicación } \\
\text { asintoticica } \\
\text { (bilateral) } \\
\end{array}$ \\
\hline Chi-cuadrado de Pearson & & 0,036 \\
\hline
\end{tabular}

Fuente: Elaboración propia, sobre Encuesta GEM 2014.

En otros clústeres se encuentran países donde los análisis muestran igualmente diferencias significativas en las elecciones de hombres y mujeres en cuanto a la financiación del emprendimiento. Así, dentro del clúster 5, en Australia se contrasta una situación similar a la de Sudáfrica: las mujeres declaran más acudir a ahorros personales $(68,1 \%$, frente al $57,6 \%$ de los hombres) y menos a bancos (14,5\%, frente al $25 \%$ de los hombres). Por su parte, la respuesta sobre preferencia de otros fondos, muestra una mayor relevancia entre hombres $(14,1 \%)$ que entre mujeres $(4,3 \%)$, de forma análoga a lo contrastado en España. 
Tabla. 7. Fuentes financiación declaradas por emprendedores, Australia

\begin{tabular}{|c|c|c|}
\hline & Hombres & Mujeres \\
\hline Ahorros Personales & $57,6 \%$ & $68,1 \%$ \\
\hline Ahorros Familia & $1,1 \%$ & $11,6 \%$ \\
\hline $\begin{array}{l}\text { Bancos y otras } \\
\text { Instituciones Fin. }\end{array}$ & $25,0 \%$ & $14,5 \%$ \\
\hline Amigos & $2,2 \%$ & $1,4 \%$ \\
\hline Otros & $14,1 \%$ & $4,3 \%$ \\
\hline Total & $100,0 \%$ & $100,0 \%$ \\
\hline \multicolumn{3}{|c|}{ Pruebas de chi-cuadrado } \\
\hline \multirow[b]{2}{*}{ Chi-cuadrado de Pearson } & \multirow[t]{2}{*}{ df } & $\begin{array}{l}\text { Significación } \\
\text { asintótica } \\
\text { (bilateral) }\end{array}$ \\
\hline & & 0,006 \\
\hline
\end{tabular}

Fuente: Elaboración propia, sobre Encuesta GEM 2014.

Desde una perspectiva de desarrollo financiero, los datos del Banco Mundial (World Bank, 2018) de estos tres países muestran que son países con un alto nivel de crédito doméstico al sector privado en relación a su PIB en 2014: España con un 129,9\%; Australia con un 128,7\%; y Sudáfrica con un 151,1\%. Esto abre nuevas cuestiones sobre la influencia del sector financiero en las expectativas de las emprendedoras frente a los emprendedores en países con un sector doméstico muy apalancado.

También dentro del clúster 1, Canadá (Tabla 8) cumple esta pauta de diferencias en las preferencias de hombres y mujeres. Así, las emprendedoras confían más que los hombres en la financiación de bancos $(28,6 \%)$ y ahorros de sus familias $(10,7 \%)$. Los hombres prefieren fundamentalmente contar para sus negocios con sus propios ahorros $(64,1 \%$, frente al $48,8 \%$ de las mujeres), seguido por Bancos $(15,9 \%)$. Al igual que en España y Australia, encontramos importantes diferencias en las respuestas de otras fuentes de financiación dados por hombres y mujeres: una mayor relevancia entre hombres $(15,2 \%)$ que entre mujeres $(9,5 \%)$. 
Tabla. 8. Fuentes financiación declaradas por emprendedores, Canadá

\begin{tabular}{|l|r|r|}
\hline & Hombres & Mujeres \\
\hline Ahorros Personales & $64,1 \%$ & $48,8 \%$ \\
\hline Ahorros Familia & $3,4 \%$ & $10,7 \%$ \\
\hline $\begin{array}{l}\text { Bancos y otras } \\
\text { Instituciones Fin. }\end{array}$ & $15,9 \%$ & $28,6 \%$ \\
\hline Amigos & $1,4 \%$ & $2,4 \%$ \\
\hline Otros & $15,2 \%$ & $9,5 \%$ \\
\hline Total & $100,0 \%$ & $100,0 \%$ \\
\hline & Pruebas de chi-cuadrado & \\
\hline & & \\
\hline
\end{tabular}

Fuente: Elaboración propia, sobre Encuesta GEM 2014.

Sin embargo, en otros países los datos muestran conclusiones diferentes, donde las pruebas de chi-cuadrado a nivel local no permite rechazar la hipótesis 3, es decir emprendedoras y emprendedores declaran acudir a fuentes similares de financiación. Dentro del clúster 3, hay varios países donde ocurre así. Es el caso de Estados Unidos (Tabla 9), donde ambos grupos confían de forma similar en ahorros personales y financiación por bancos. Además, en este país, encontramos una importante diferencia respecto los anteriores países: una cierta proximidad en términos de otras fuentes de financiación: $25 \%$ para los hombres y el 19\% para las mujeres.

Tabla. 9. Fuentes financiación declaradas por emprendedores, EE.UU.

\begin{tabular}{|l|r|r|}
\hline & Hombres & Mujeres \\
\hline Ahorros Personales & $45,4 \%$ & $49,6 \%$ \\
\hline Ahorros Familia & $3,6 \%$ & $9,1 \%$ \\
\hline $\begin{array}{l}\text { Bancos y otras } \\
\text { Instituciones Fin. }\end{array}$ & $23,0 \%$ & $21,5 \%$ \\
\hline Amigos & $3,1 \%$ & $0,8 \%$ \\
\hline Otros & $25,0 \%$ & $19,0 \%$ \\
\hline Total & $100,0 \%$ & $100,0 \%$ \\
\hline
\end{tabular}

Fuente: Elaboración propia, sobre Encuesta GEM 2014. 
En México (Tabla 10), ambos grupos también confían de forma similar en tres fuentes: ahorros personales, ahorros de la familia y préstamos personales. En varios países del clúster 3 se repite esta similitud entre las respuestas declaradas por hombres y mujeres, como Rusia, Alemania y Brasil.

Tabla. 10. Fuentes financiación declaradas por emprendedores, México.

\begin{tabular}{|c|c|c|}
\hline & Hombres & Mujeres \\
\hline Ahorros Personales & $51,1 \%$ & $50,6 \%$ \\
\hline Ahorros Familia & $21,3 \%$ & $26,8 \%$ \\
\hline $\begin{array}{l}\text { Bancos y otras } \\
\text { Instituciones Fin. }\end{array}$ & $21,8 \%$ & $19,0 \%$ \\
\hline Amigos & $2,7 \%$ & $2,4 \%$ \\
\hline Otros & $3,2 \%$ & $1,2 \%$ \\
\hline Total & $100,0 \%$ & $100,0 \%$ \\
\hline \multicolumn{3}{|c|}{ Pruebas de chi-cuadrado } \\
\hline Valor & df & $\begin{array}{c}\text { Significación } \\
\text { asintotica } \\
\text { (bilateral) }\end{array}$ \\
\hline
\end{tabular}

Fuente: Elaboración propia, sobre Encuesta GEM 2014.

Igualmente, encontramos países en los diferentes clústeres donde contrastamos resultados similares entre emprendedores de ambos géneros, y por tanto donde los datos permiten rechazar la hipótesis 3. En el clúster 2, sería el caso de Perú (Tabla 11), donde ambos grupos confían ampliamente en la financiación ajena, tanto de bancos como de familiares. 
Tabla. 11. Fuentes financiación declaradas por emprendedores, Perú.

\begin{tabular}{|c|c|c|}
\hline & Hombres & Mujeres \\
\hline Ahorros Personales & $37,0 \%$ & $39,6 \%$ \\
\hline Ahorros Familia & $17,8 \%$ & $21,6 \%$ \\
\hline $\begin{array}{l}\text { Bancos y otras } \\
\text { Instituciones Fin. }\end{array}$ & $44,2 \%$ & $38,4 \%$ \\
\hline Amigos & $0,4 \%$ & $0,0 \%$ \\
\hline Otros & $0,7 \%$ & $0,4 \%$ \\
\hline Total & $100,0 \%$ & $100,0 \%$ \\
\hline \multicolumn{3}{|c|}{ Pruebas de chi-cuadrado } \\
\hline \multirow[b]{2}{*}{ Chi-cuadrado de Pearson } & df & $\begin{array}{c}\text { Signnificación } \\
\text { asintotica } \\
\text { (bilateralal) }\end{array}$ \\
\hline & $3,445^{\mathrm{a}}$ & 0,486 \\
\hline
\end{tabular}

Fuente: Elaboración propia, sobre Encuesta GEM 2014.

En el clúster 4, encontramos a Italia (Tabla 12), y también hay similitudes entre ambos grupos en otros países, como India. Salvo el caso de Estados Unidos, el resto de los países donde se identificaban similitudes entre hombres y mujeres coinciden con países donde el crédito doméstico al sector privado era en 2014 inferior al 100\% de su PIB según el Banco Mundial: México 29,3\%; Rusia, 53,5\%; Alemania, 79,2\%; Brasil, 66,0\%; Italia, 88,9\%; India, 51,9\%. 
Tabla. 12. Fuentes financiación declaradas por emprendedores, Italia.

\begin{tabular}{|c|c|c|}
\hline & Hombres & Mujeres \\
\hline Ahorros Personales & $48,2 \%$ & $59,3 \%$ \\
\hline Ahorros Familia & $10,9 \%$ & $13,0 \%$ \\
\hline $\begin{array}{l}\text { Bancos y otras } \\
\text { Instituciones Fin. }\end{array}$ & $20,9 \%$ & $14,8 \%$ \\
\hline Amigos & $3,6 \%$ & $1,9 \%$ \\
\hline Otros & $16,4 \%$ & $11,1 \%$ \\
\hline Total & $100,0 \%$ & $100,0 \%$ \\
\hline \multicolumn{3}{|c|}{ Pruebas de chi-cuadrado } \\
\hline \multirow[b]{2}{*}{ Chi-cuadrado de Pearson } & df & $\begin{array}{c}\text { Significación } \\
\text { asintótica } \\
\text { (bilateral) }\end{array}$ \\
\hline & $2,762^{\mathrm{a}}$ & 0,598 \\
\hline
\end{tabular}

Fuente: Elaboración propia, sobre Encuesta GEM 2014.

En definitiva, se identifican diversos países donde las preferencias declaradas por los emprendedores son similares entre hombres y mujeres, como ocurre en Estados Unidos, a diferencia de los que ocurre en países como España, Canadá o Australia. Esto abre importantes cuestiones para futuros estudios sobre las variables que determinan las preferencias de las emprendedoras en cuanto a las fuentes de financiación de sus proyectos empresariales.

\section{Conclusiones}

Este estudio ha utilizado en un primer momento un planteamiento teórico que explica el estado de la cuestión del emprendimiento femenino desde el punto de vista de los procesos financiadores del mismo para determinar, entre otras cosas, prácticas discriminatorias de género en el acceso a la financiación social, así como las preferencias financieras en la puesta en marcha de negocios liderados por mujeres. Con base en el último informe disponible del GEM, un primer análisis arroja que dentro de cada país, en términos de género, no se puede contrastar que las mujeres necesiten menos dinero que los hombres. Este resultado difiere de estudios anteriores (Marlow y Patton, 2005; Coleman y Robb, 2009), y abre posibles líneas de investigación sobre cómo satisfacen las emprendedoras sus necesidades de financiación.

El estudio relativo a las fuentes de financiación declaradas por las emprendedoras de 69 países confirma la relevancia del contexto nacional de la emprendedora. Se han identificado 5 clústeres con perfiles diferentes en términos de las preferencias declaradas por las emprendedoras. Las variables que más 
discriminan son relativas al acceso a financiación social ajena, bien mediante bancos o bien mediante sus propios familiares. Por esto, es importante estudiar en profundidad la demanda de financiación formal de las entidades financieras, y como éstas la atienden desde la oferta. Pero también las diferencias locales afectan a unas crecientes fuentes de financiación para el emprendimiento, como venture capital o business angels. Estas fuentes están ya muy presentes en países como Estados Unidos, pero no tanto en otros como España.

Finalmente, el contraste de género entre las fuentes de financiación declaradas por los emprendedores cuestiona nuevamente que las mujeres necesariamente prefieran financiar su negocio con fuentes diferentes a las de los hombres. Por una parte, tanto de una forma agregada como a nivel local en países como España, Canadá o Australia, las emprendedoras declaran fuentes diferentes a las de sus homólogos masculinos. Estos resultados estarían alineados con diversos estudios (Sena et al., 2012; Watson et al. 2017). Pero los estudios locales de países como Estados Unidos, México, Alemania o Italia muestran conclusiones diferentes: no podemos afirmar en estos países que el género esté asociado a diferencias en las fuentes de financiación preferidas.

Estos análisis de la demanda presentan varias limitaciones. Por una parte, los datos utilizados son declarados en encuestas por los emprendedores en distintas fases del proceso, por lo que sería relevante completar este análisis contrastando los datos de la oferta de financiación en los diferentes países. Por otra parte, la diferencia contrastada en diferentes países en función del género abre cuestiones sobre las limitaciones que condicionan la demanda de financiación para el emprendimiento femenino. De esta forma, será posible dimensionar el peso del entorno local para el emprendimiento femenino, frente a variables individuales como la actitud frente al riesgo o la perspectiva de acceso a la financiación que perciben las emprendedoras. A resultas, será posible profundizar en las claves y barreras que afrontan las emprendedoras en diferentes partes del mundo.

\section{Referencias bibliográficas}

Ascher, J. (2012) Female entrepreneurship-An appropriate response to gender discrimination, Journal of Entrepreneurship, Management and Innovation, $\mathrm{N}^{\circ}$ 4, pp. 97 114.

Bates, T.; Jackson, W.E. III and Johnson, J.H. Jr (2007) Introduction to the special issue on advancing research on minority entrepreneurship, Annals of the American Academy of Political Science and Social Science, Vol. 613, pp. 10-17.

Becker-Blease, J. R.; Sohl, J. E. (2007) Do women-owned businesses have equal access to angel capital? Journal of Business Venturing, No 22, Vol. 4, pp. 503-521.

Bosse, Douglas A., and Porcher L. Taylor, III (2012) The Second Glass Ceiling Impedes Women Entrepreneurs, The Journal of Applied Management and Entrepreneurship 17, $\mathrm{N}^{\mathrm{o}}$ 1, pp. 52-68.

Brush, C., Bruin, A. y Welter, F. (2009) A gender-aware framework for women's entrepreneurship, International Journal of Gender and Entrepreneurship, $\mathrm{N}^{\mathrm{o}} 1$, pp. 824. 
Brush, C., Carter, N., Gatewood, E., Greene, P. and Hart, M. (2006) Gatekeepers of venture growth: The role and participation of women in the venture capital industry, The Kauffman Foundation.

Buckland, L. y Cordobés, M. (2017) Inversión con enfoque de género: una oportunidad para la empresa social Europe, ESADE, Madrid.

Carter, S., Shaw, E., Wilson, F. and Lam, W. (2007) Gender, entrepreneurship and bank lending: the criteria and processes used by bank loan officers in assessing applications, Entrepreneurship Theory \& Practice, № 31, Vol. 3, pp. 427-444.

Cohoon, J.M., Wadhwa, V. and Mitchell, L. (2010) The anatomy of an entrepreneur: Are successful women entrepreneurs different from men? The Kauffman Foundation.

Cotter, D. A., Hermsen, J. M., Ovadia, S. y Vanneman, R. (2001) The glass ceiling effect, Social forces, № 80, Vol. 2, pp. 655-681.

Coleman, S., Cotei, C. y Farhat, J. (2016) The debt-equity financing decisions of US startup firms, Journal of Economics and Finance, $\mathrm{N}^{\circ}$ 40, Vol. 1, pp. 105-126.

Coleman, S. y Robb, A. (2009) A comparison of new firm financing by gender: evidence from the Kauffman Firm Survey data, Small Business Economics, No 33, Vol. 4, p. 397.

European Parliament (2015) Women's Entrepreneurship: Closing the gender gap in access to financial and other services and in social entrepreneurship, Directorate General for Internal Policies.

Global Entrepreneurship Monitor (2017) Global Entrepreneurship Monitor 2016/2017. Report on Women's Entrepreneurship, Global Entrepreneurship Research Association.

Harrison, R. T. y Mason, C. M. (2007) Does gender matter? Women business angels and the supply of entrepreneurial finance, Entrepreneurship Theory and Practice, $\mathrm{N}^{\circ} 31$, Vol. 3, pp. 445-472.

Henry, C., Foss, L. y Ahl, H. (2016) Gender and entrepreneurship research: A review of methodological approaches. International Small Business Journal, $\mathrm{N}^{\circ} 34$, Vol. 3, pp. 217-241.

Kollmann, T. y Kuckertz, A. (2010) Evaluation uncertainty of venture capitalists' investment criteria, Journal of Business Research, № 63, pp. 741-747.

Kwong, C., Jones-Evans, D. y Thompson, P. (2012) Differences in perceptions of access to finance between potential male and female entrepreneurs: Evidence from the UK, International Journal of Entrepreneurial Behavior \& Research, $\mathrm{N}^{\mathrm{o}}$ 18, Vol. 1, pp. 7597.

Magri, S. (2009) The financing of small innovative firms: the Italian case, Economics of Innovation and New Technology, $\mathrm{N}^{\mathrm{o}}$ 18, Vol. 2, pp. 181-204.

Marlow, S. y Patton, D. (2005) All credit to men? Entrepreneurship, finance, and gender, Entrepreneurship theory and practice, $\mathrm{N}^{\circ} 29$, Vol. 6, pp. 717-735.

Marlow, S. y Swail, J. (2014) Gender, risk and finance: why can't a woman be more like a man?, Entrepreneurship \& Regional Development, № 26, Vol. 1-2, pp. 80-96.

Marom, D., Robb, A. y Sade, O. (2016) Gender dynamics in crowdfunding (Kickstarter): Evidence on entrepreneurs, investors, deals and taste-based discrimination.

Montero González, B. y Camacho Ballesta, J.A. (2018) Caracterización del emprendimiento femenino en España: Una visión de conjunto, REVESCO. Revista de Estudios Cooperativos, Tercer Cuatrimestre, No 129. DOI: 10.5209/REVE.61936.

Myers, S. C. (1984) The capital structure puzzle, The journal of finance, $\mathrm{N}^{\mathrm{o}} 39$, Vol. 3, pp. 574-592.

OECD (2017) Entrepreneurship at a Glance 2017, OECD Publishing.

Orser, B. J., Riding, A. L. y Manley, K. (2006) Women entrepreneurs and financial capital, Entrepreneurship Theory and Practice, No 30, Vol. 5, pp. 643-665. 
Poggesi, S., Mari, M. y De Vita, L. (2016) What's new in female entrepreneurship research? Answers from the literature, International Entrepreneurship and Management Journal, $\mathrm{N}^{\mathrm{o}} 12$, Vol. 3, pp. 735-764.

Roper, S. \& Scott, J. M. (2009) Perceived financial barriers and the start-up decision: An econometric analysis of gender differences using GEM data, International Small Business Journal, № 27, Vol. 2, pp. 149-171.

Sánchez Espada, J., Martín López, S., Bel Durán, P. y Lejarriaga Pérez de las Vacas, G. (2018) Educación y formación en emprendimiento social: características y creación de valor social sostenible en proyectos de emprendimiento social. REVESCO. Revista de Estudios Cooperativos, Tercer Cuatrimestre, $\mathrm{N}^{\circ} 129$, pp. 16-38. DOI: 10.5209/REVE.62492.

Schoon, I. y Duckworth, K. (2012) Who becomes an entrepreneur? Early life experiences as predictors of entrepreneurship, Developmental psychology, $\mathrm{N}^{\circ}$ 48, Vol. 6, p. 1719.

Sena, V., Scott, J. y Roper, S. (2012) Gender borrowing and self-employment: Some evidence for England, Small Business Economy, № 38, pp. 467-480.

Shane, S. y Cable, D. (2002) Network ties, reputation, and the financing of new ventures, Management Science, $\mathrm{N}^{\circ} 48$, pp. 364-381

Verheul, I. y Thurik, R. (2001) Start-up capital: does gender matter?, Small business economics, $\mathrm{N}^{\circ} 16$, Vol. 4, pp. 329-346.

Villaseca, D. (2014) Innovación y Marketing de Servicios en la Era Digital, ESIC Editorial.

Watson, J., Stuetzer, M. y Zolin, R. (2017) Gender Differences in New Venture Funding, The Routledge Companion to Global Female Entrepreneurship.

World Bank (2018), World Development Indicators, World Bank.

Xiong, X., Fu, R., Zhang, W., Zhang, Y. y Xiong, L. (2011) The research on the influencing factors of financing strategy of woman entrepreneurs in China, JCP, $\mathrm{N}^{\circ} 6$, Vol. 9, pp. 1819-1824. 